\title{
Extrasolar planet interactions
}

\author{
Rory Barnes ${ }^{1}$ and Richard Greenberg ${ }^{1}$ \\ ${ }^{1}$ Lunar and Planetary Lab, University of Arizona, 1629 E. University Blvd., Tucson, AZ, USA \\ email: rory@lpl.arizona.edu
}

\begin{abstract}
The dynamical interactions of planetary systems may be a clue to their formation histories. Therefore, the distribution of these interactions provides important constraints on models of planet formation. We focus on each system's apsidal motion and proximity to dynamical instability. Although only $\sim 25$ multiple planet systems have been discovered to date, our analyses in these terms have revealed several important features of planetary interactions. 1) Many systems interact such that they are near the boundary between stability and instability. 2) Planets tend to form such that at least one planet's eccentricity periodically drops to near zero. 3) Mean-motion resonant pairs would be unstable if not for the resonance. 4) Scattering of approximately equal mass planets is unlikely to produce the observed distribution of apsidal behavior. 5) Resonant interactions may be identified through calculating a system's proximity to instability, regardless of knowledge of angles such as mean longitude and longitude of periastron (e.g. GJ $317 \mathrm{~b}$ and c are probably in a 4:1 resonance). These properties of planetary systems have been identified through calculation of two parameters that describe the interaction. The apsidal interaction can be quantified by determining how close a planet is to an apsidal separatrix (a boundary between qualitatively different types of apsidal oscillations, e.g. libration or circulation of the major axes). This value can be calculated through short numerical integrations. The proximity to instability can be measured by comparing the observed orbital elements to an analytic boundary that describes a type of stability known as Hill stability. We have set up a website dedicated to presenting the most up-to-date information on dynamical interactions: http://www.lpl.arizona.edu/ rory/research/xsp/dynamics.
\end{abstract}

\section{Introduction}

One of the most striking differences between known exoplanets and the giant planets of our Solar System involves the observed orbits: Observed exoplanets tend to have large eccentricities $e$, and small semi-major axes $a$, whereas the gas giants of the Solar System have small $e$ and large $a$. Recently, however, it has been shown that the dynamical interactions in many multiple planet systems (including the Solar System) show certain features in common (Barnes \& Quinn 2004; Barnes \& Greenberg 2006a [BG06a]; Barnes \& Greenberg 2006c [BG06c]; Barnes \& Greenberg 2007b [BG07b]). These shared traits suggest that the character of dynamical interactions (over $10^{3}-10^{4}$ years), rather than the present orbits, may be a more meaningful constraint on the origins of planetary systems (Barnes \& Greenberg 2007a [BG07a], BG07b). Considerations of shared dynamical properties have even resulted in the first successful prediction of the mass and orbit of an extrasolar planet, HD 74156 d (predicted by Barnes \& Raymond [2004] and Raymond \& Barnes [2005]; found by Bean et al.2008).

Now with over 200 extra-solar planets known, including $\sim 25$ multi-planet systems, we can look at this population as a whole, with increasing confidence regarding which common characteristics may be more than statistical flukes. We have identified commonalities among planetary interactions that may be key constraints on the origins of planetary systems. We have identified parameters that quantify an interaction's prox- 
imity to boundaries between qualitatively different types of motion. These two types of boundaries are the "apsidal separatrix" and the dynamical stability boundary. The apsidal separatrix is the boundary between different types of apsidal oscillations, e.g. libration and circulation. The stability boundary separates regions in which all planets are bound to the host star from those in which at least one planet is liable to be ejected. These two parameters are fixed quantities that do not vary over time, but they constrain how the systems evolve. Systems tend to lie close to these two boundaries.

In this chapter we review our derivation of $\epsilon$, which quantifies proximity to an apsidal separatrix, in $\S 2$. Then we describe $\beta$, which parameterizes a system's proximity to dynamical instability, in $\S 3$. In $\S 4$ we present the observed distributions of these quantities and use them to constrain formation models. Finally in $\S 5$ we draw our general conclusions.

\section{The Apsidal Separatrix}

Eccentricities in multiple planet systems oscillate due to secular interactions (see e.g. Laughlin \& Adams 1999; Rivera \& Lissauer 2000; Stepinski et al.2000; Michtchenko \& Malhotra 2002; BG06a). Thus a currently observed eccentricity may not be its average value over the $\sim 10,000$ year secular period. Validation of formation models through the eccentricity distribution is therefore inadequate; the eccentricity oscillations are a better description of a multiple-planet system's properties.

We consider analytic and numerical models of planet-planet interactions to describe the oscillations. The analytic approach is secular theory which considers long-term averages of forces between planets. This method was originally developed independently by Laplace and Lagrange, but see BG06a for a presentation that does not involve matrix manipulation. We compare the secular solutions to $N$-body numerical models that solve the force equations directly, and are therefore arbitrarily accurate, for a sufficiently small timestep, using the MERCURY6 code (Chambers et al.1999).

In general, secular theory predicts that $e$ 's and $\Delta \varpi$ 's (the difference between two longitudes of periastron, $\varpi$ ) oscillate and that $a$ is constant. Therefore, conservation of angular momentum requires that as one planet's eccentricity drops, another must rise (as $e$ increases, orbital angular momentum decreases). The type of oscillation depends on initial conditions. If $\Delta \varpi$ oscillates about 0 , the system is experiencing "aligned libration." If $\Delta \varpi$ librates about $\pi$, then the system is undergoing "anti-aligned libration." If $\Delta \varpi$ oscillates through $2 \pi$ then the apsides undergo "circulation". The boundaries between these qualitatively different types of behavior are known as "separatrices".

Recently it has been noted that many systems lie near an "apsidal separatrix" (Ford et al.2005; BG06a, BG06c). For systems of just two planets, the apsidal separatrix can only separate circulation and libration. This type of separatrix is a "libration-circulation separatrix". An example of the libration-circulation separatrix is shown in the left panels of Fig. 1.

In systems of more than two planets, things get more complicated. In addition to the libration-circulation separatrix, the system may interact with different numbers of rotations of $\Delta \varpi$ through $360^{\circ}$ during one eccentricity oscillation. The boundary between interactions with different numbers of circulations in one eccentricity cycle is a "circulation-mode separatrix", and an example is shown in the right panels of Fig. 1. Note that the gray curve in the top right panel of this figure circulates once and librates once during one eccentricity cycle, whereas the black curve circulates twice.

For an interaction to lie near a separatrix, the amplitude of eccentricity oscillations is generally two orders of magnitude or more. Since $0 \leqslant e<1$ for bound planets, this 

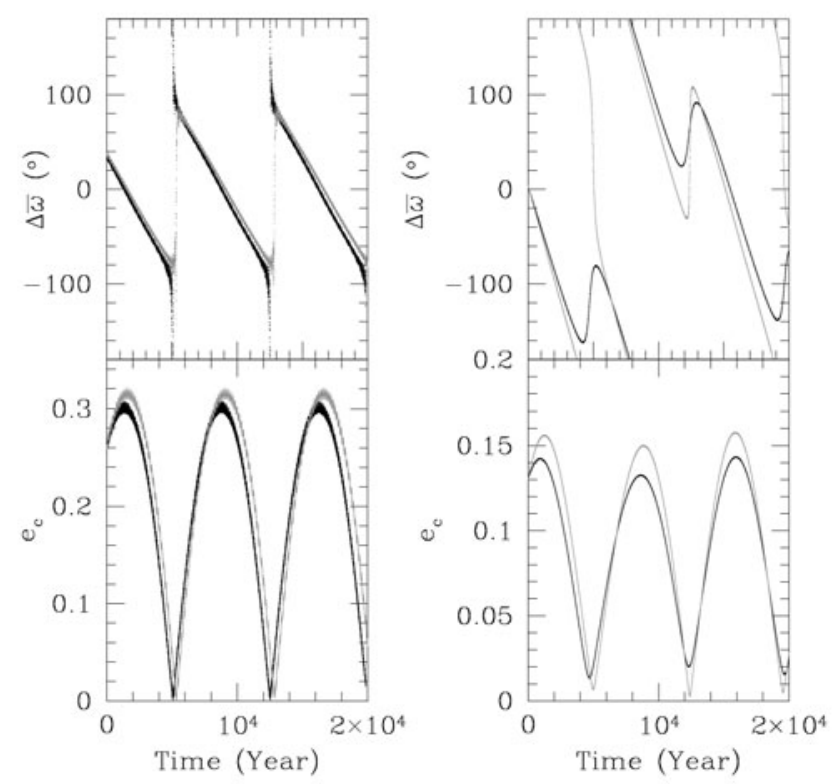

Figure 1. Examples of near-separatrix motion in planetary systems. Left Panels: A libration-circulation separatrix. Two possible evolutions of $v$ And $\mathrm{c}$ and $\mathrm{d}$ (the middle and outer planet of the system) assuming the current orbits. The black points are the system from Butler et al.(2006), the gray from Ford et al.(2005). Although the best-fit orbits in these two cases are very similar, they result in qualitatively different types of evolution of $\Delta \varpi$ (top): The older data predict aligned libration, whereas the updated data predict circulation. Note that the evolution of $e_{c}$ is similar in both cases, and periodically reaches near-zero values (bottom). Right Panels: A circulation-mode separatrix. HD $69830 \mathrm{c}$ and d evolve near the circulation-mode separatrix. The black data are from Lovis et al.(2006), and the gray data are for a fictitious system in which the inner planet's, b's, eccentricity was changed from 0.1 to 0.15 . In the first $2 \times 10^{4}$ years, the actual $\Delta \varpi$ undergoes 1 complete rotation through $360^{\circ}$, but in the fictitious system, $\Delta \varpi$ undergoes 2 complete circulations (top). We again see that the middle planet's eccentricity periodically drops to near-zero values (bottom).

means that at least one planet in near-separatrix interactions (both libration-circulation and circulation-mode) periodically is on a nearly circular orbit. The proximity to the separatrix can be parameterized as

$$
\epsilon \equiv \frac{2\left[\min \left(\sqrt{x^{2}+y^{2}}\right)\right]}{\left(x_{\max }-x_{\min }\right)+\left(y_{\max }-y_{\min }\right)},
$$

where $x \equiv e_{1} e_{2} \sin (\Delta \varpi) ; y \equiv e_{1} e_{2} \cos (\Delta \varpi)$, and the subscripts min and max refer to the extreme values these variables attain over a complete secular cycle (BG06c). (Note that $\epsilon$ is a fixed quantity even as $e$ and $\Delta \varpi$ change periodically.) When $\epsilon=0$ the pair is on an apsidal separatrix, and one eccentricity periodically reaches zero. Note that in the case of mean motion resonant interactions, $\epsilon$ may not necessarily reveal how close an interaction is to an apsidal separatrix (BG06c).

\section{The Dynamical Stability Limit}

Observational uncertainties in the orbits of exoplanets often include regions of dynamical instability in which one or more planets would be ejected within $1 \mathrm{Myr}$, i.e. the system is dynamically "packed" (e.g. Barnes \& Quinn 2001, 2004; Goździewski 2002; 
Kiseleva-Eggleton 2002; Érdi et al.2004; BG06b, BG07b). This timescale appears to be long enough to identify nearly all unstable configurations (Barnes \& Quinn 2004). Representative cases of these investigations are shown in Fig. 2. The shading in this figure correlates with the likelihood of stability: White regions are stable, black unstable, and grays contain a mix of stable and unstable orbits. In these cases we define stability in the "Lagrange" sense for $\sim 10^{6}$ orbits: Escapes and exchanges of planets are forbidden.

The preponderance of systems that lie near the stability limit naturally raises the question of where, exactly, in orbital element space, the stability boundary lies. Surprisingly, a general analytical expression for the location of the stability boundary is lacking, even though it has been the goal of considerable research (e.g. Szebehely \& McKenzie 1981; Wisdom 1982; Marchal \& Bozis 1982; Gladman 1993; Laskar 1990; Holman \& Wiegert 1999; Sosnitskii 1999; David et al.2003; Cuntz et al.2007). These investigations all made significant progress toward defining a stability boundary, but none found it.

Given that many planetary systems lie near the dynamical stability limit, we have considered how to quantify the proximity to stability (BG06b, BG07b). We will focus on one formulation by Marchal \& Bozis (1982; see also Gladman 1993) that considers "Hill stability". Hill stability only requires the ordering of the planets to remain constant for all time; the outer planet may escape to infinity.

A coplanar, two-planet system, outside of a resonance is Hill stable if

$$
-\frac{2 M}{G^{2} M_{*}^{3}} c^{2} h>1+3^{4 / 3} \frac{m_{1} m_{2}}{m_{3}^{2 / 3}\left(m_{1}+m_{2}\right)^{4 / 3}}-\frac{m_{1} m_{2}\left(11 m_{1}+7 m_{2}\right)}{3 m_{3}\left(m_{1}+m_{2}\right)^{2}}+\ldots,
$$

where $M$ is the total mass of the system, $m_{1}$ is the mass of the more massive planet, $m_{2}$ is the mass of the less massive planet, $m_{3}$ is the mass of the star, $G$ is the gravitational constant, $M_{*}=m_{1} m_{2}+m_{1} m_{3}+m_{2} m_{3}, c$ is the total angular momentum of the system, and $h$ is the energy (Marchal \& Bozis 1982). Here $c$ and $h$ must be calculated in barycentric coordinates. If a given three-body system satisfies the inequality in Eq. (3.1), then the system is Hill stable. If this inequality is not satisfied, then the system may or may not be Hill stable. In this inequality, the left-hand side is a function of the orbits, but the right-hand side is only a function of the masses. This approach for identifying stable orbits is fundamentally different from other common techniques for determining stability which exploit resonance overlaps (Wisdom 1982; Quillen \& Faber 2006), chaotic diffusion (Laskar 1990; Pepe et al.2007), fast Lyapunov indicators (Froeschlé et al.1997; Sándor et al.2007), or periodic orbits (Voyatzis \& Hadjidemetriou 2006; Hadjidemetriou 2006).

The ratio of the two sides of the inequality in Eq. (3.1) (left-hand side over right-hand side) allows a quantification of a system's proximity to the Hill stability boundary. We define this ratio as $\beta$. If $\beta<1$ a system's Hill stability is unknown, if $\beta>1$, Hill stability is guaranteed, if $\beta=1$, the system is on the boundary. Note that for any system of two planets outside of resonance with fixed mass, the locus of Hill-stable orbits can be easily calculated.

Fig. 2 includes contour lines of constant $\beta$ for the four systems considered. For the bottom two cases (systems with no mean motion resonance), the $\beta=1$ lines are near the transition from Lagrange stable orbits (white) to unstable (black). These two cases therefore show that when $\beta \gtrsim 1$, a system is not only Hill stable, it is also Lagrange stable.

However, in the top two panels of Fig. 2 , the $\beta=1$ contour cuts through a swath of stability located at resonance. This qualitatively different behavior is no surprise because the criterion for Hill stability, Eq. (3.1), does not apply to systems in resonance. Note however that in the top left panel, outside of resonance, the $\beta$ contours do follow the 

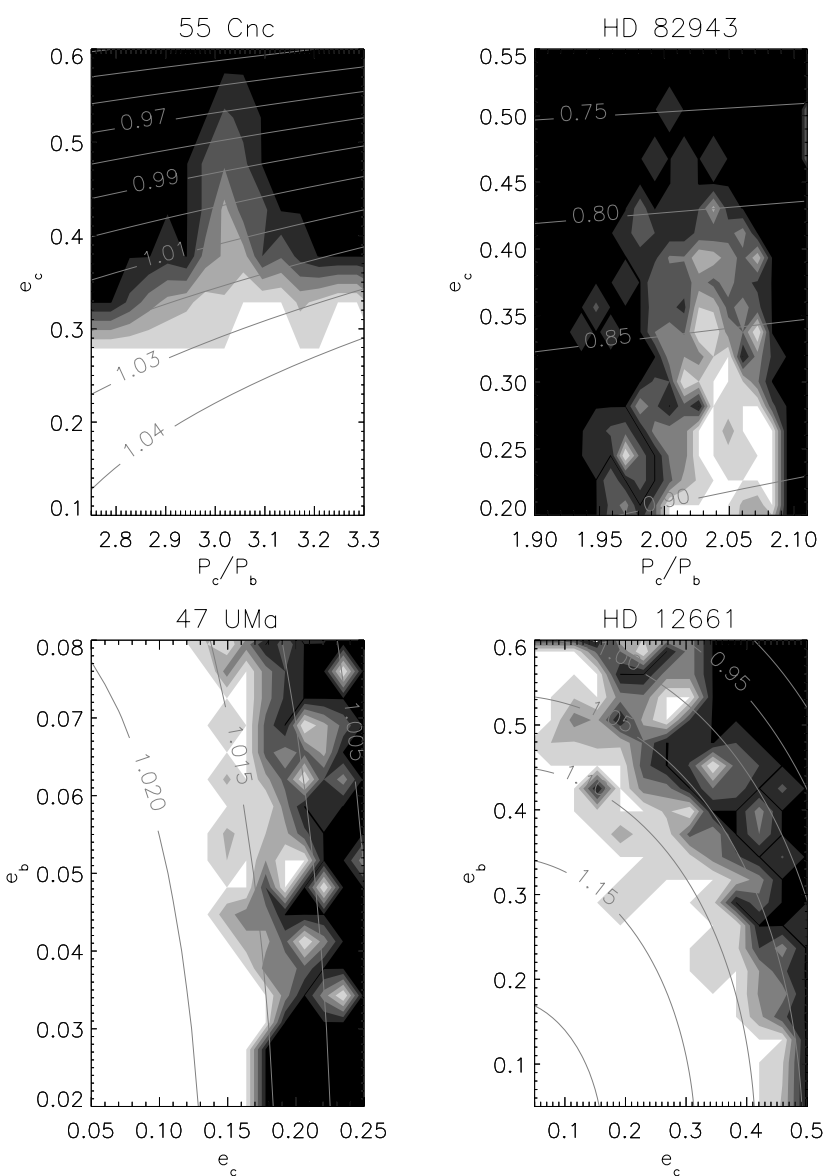

Figure 2. Lagrange stability boundary in relation to the Hill stability boundary for four exoplanetary systems. White regions represent bins in which all configurations were stable, black bins contained no stable configurations, darker shades of gray correspond to regions in which the fraction of stable simulations were smaller (e.g. Barnes \& Quinn 2004). The curves represent contour lines of $\beta$. Contour lines follow the shape of the Lagrange stability boundary, except in resonance, where the Lagrange stability region is extended to lower values of $\beta$.

Lagrange stability boundary, again at values near 1 . In the top right, we see that all $\beta$ values are less than 1 , hence there are no non-resonant, Lagrange stable configurations in the region of orbital element space considered.

\section{Dynamical Properties of Exoplanets}

In this section we will tabulate the observed distributions of $\epsilon$ and $\beta$ and interpret these distributions in the context of models of planet formation. Observed extrasolar planets tend to lie near the apsidal separatrix. In the top panel of Fig. 3, we plot the observed distribution of $\epsilon$ values (solid line). These values were calculated through an $N$ body integration that required energy conservation to be better than 1 part in $10^{4}$ with a symplectic integration technique. Over $40 \%$ of known systems have $\epsilon<0.01$, including two of the three giant-planet pairs in the Solar System. 
We tabulate the dynamical properties of multi-planet systems in Table $1 \dagger$, where we also list the sources of the initial conditions we used. In this table AM stands for "apsidal motion" and the possibilities are circulation (C), aligned libration (A), or anti-aligned libration (AA). The MMR column lists the resonance, if applicable. The proximities to the apsidal separatrix, $\epsilon$, are the values from the literature (BG06c), as are proximities to the Hill stability boundary, $\beta$ (BG07b). The "Class" distinguishes orbits whose evolution is dominated by tidal $(\mathrm{T})$, resonant $(\mathrm{R})$ or secular $(\mathrm{S})$ interactions. Table 1 includes the dynamical properties of the giant planets in our Solar System for comparison.

Given the strong tendency for systems to be near the apsidal separatrix, it is natural to wonder how systems formed that way. The apsidal behavior is tied to the eccentricities. Considerable work by previous researchers has focused on reproducing the distribution of eccentricity values. The two most studied processes are "planet-planet scattering", i.e. gravitational encounters between planets (e.g. Rasio \& Ford 1996; Weidenschilling \& Marzari 1996; Lin \& Ida 1997; Ford et al.2001; Marzari \& Weidenschilling 2002), and torquing by remnant protoplanetary disks that pumps up eccentricities (e.g. Artymowicz 1992; Boss 2000; Papaloizou et al.2001; Chiang \& Murray 2002; Goldreich \& Sari 2003; D'Angelo et al.2006). Simulations of planet-planet scattering can produce eccentricity distributions that are similar to the observed distribution (Ford et al.2001), but models of disk torquing tend to produce eccentricities that are too low (D'Angelo et al.2006).

Ford et al.(2005) were the first to consider how scattering may lead to near-separatrix behavior. They showed that the ejection of a hypothetical additional planet could produce the observed near-separatrix behavior among the planets orbiting $v$ And. In their model three planets, initially on circular orbits, formed too close together to be stable. Scattering between two planets results in one of them being ejected. The third planet, orbiting at a safe distance from the scattering planets, remained on a circular orbit. The planet that remained bound to the star after the ejection received an impulsive kick and its eccentricity quickly jumped to a relatively large value. This sudden change created a new "initial condition" for the secular interaction. The planet that did not partake in the scattering then began a new secular evolution, but since apsidal behavior is periodic, that planet's eccentricity would return to zero and the motion was near-separatrix.

However, Ford et al.only considered one such unstable case, and it was unclear how likely such a scenario was. The observed $\epsilon$ statistics (solid line in the top panel of Fig. 3) place a strong constraint on planet-planet scattering models. BG07a considered $\sim 400$ hypothetical systems in order to provide a statistical test of the planet-planet scattering model's likelihood to reproduce the observed apsidal behavior of extrasolar planetary systems. In the main part of that experiment, they considered systems in which all orbital elements were initially the same except for the mean longitude of the middle planet (of a three-planet system) which was shifted by 1 degree for each successive case (i.e. 360 simulations). Stability is most likely independent of this parameter (see Eq. 3.1 ), therefore there should be no correlation between initial mean longitude and $\epsilon$. The resulting distribution of $\epsilon$ is shown in the top panel of Fig. 3 by the dashed line. This scattering (dashed line) produced one-tenth the fraction of near-separatrix $(\epsilon<0.01)$ cases as is observed (solid line).

The planet-planet scattering model had done a reasonable job of reproducing the observed eccentricity distribution (Ford et al.2001), but it may be a challenge for it to reproduce the observed apsidal behavior (BG07a). BG07a noted that the path to small $\epsilon$ values seemed quite constrained: The scattered planet needed to be ejected immedi-

$\dagger$ see http://www.lpl.arizona.edu/ rory/research/xsp/dynamics for an up-to-date list of these properties 

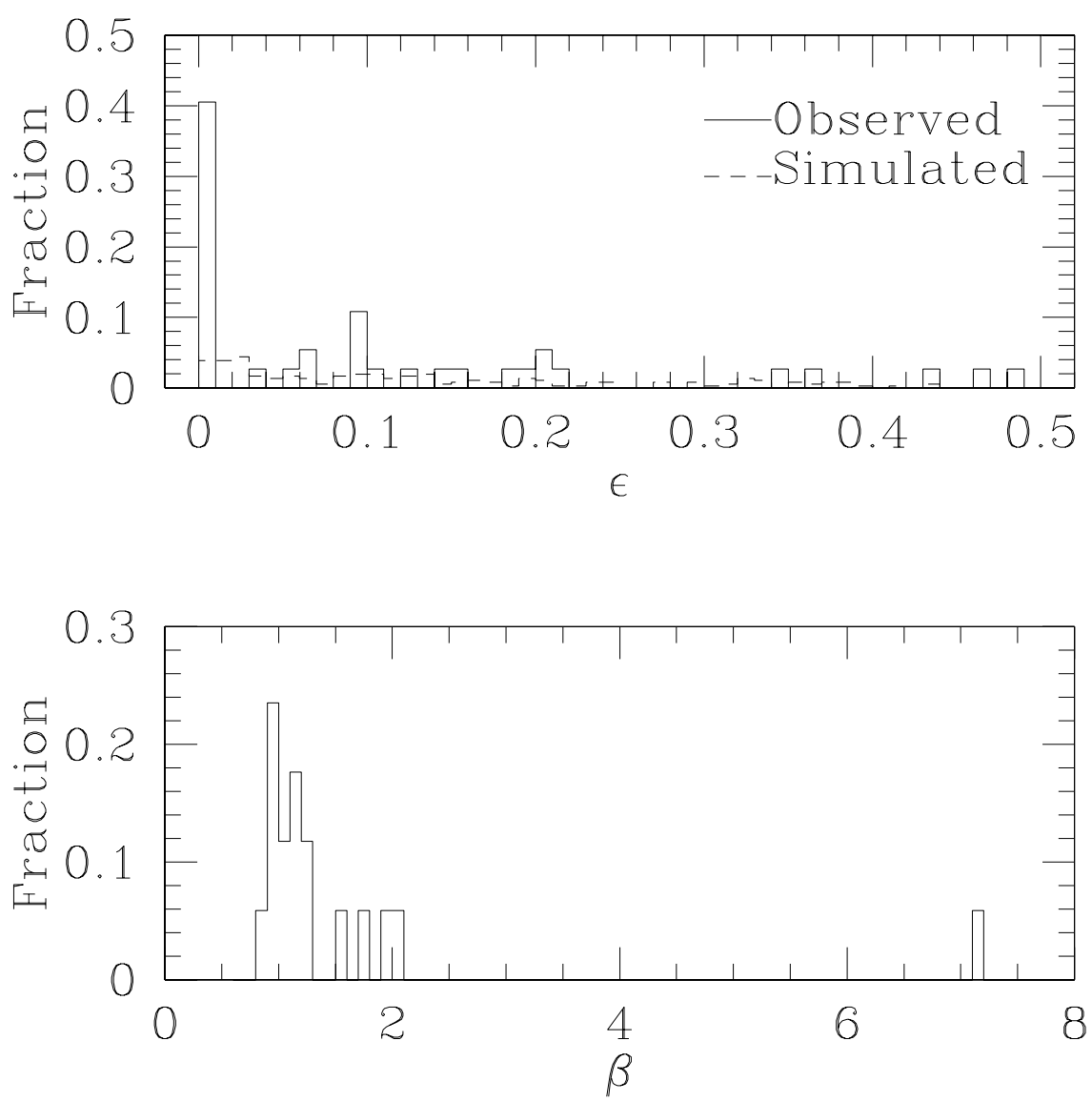

Figure 3. Top Panel: Distributions of $\epsilon$. The solid line is the observed distribution, the dashed line is that predicted by a model involving the ejection of an additional Jupiter mass planet (Ford et al.2005; BG07a). Bottom Panel: Observed distribution of $\beta$ in known two-planet systems. Note that we may only calculate $\beta$ for two-planet systems.

ately. This common scattering route to near-separatrix motion led BG07a to suggest that the probability of near-separatrix motion could be enhanced if the perturber is relatively small and/or on an initially eccentric orbit. Such a "rogue planet" may have a better chance of being removed from a planetary system after one encounter with a giant planet, but this model will require further testing. The actual distribution of $\epsilon$ must be reproduced by any model of planet formation.

Next we discuss the implications of the $\beta$ distribution, that is, how close a system is to instability. We tabulate $\beta$ (Table 1 ) values to produce another distribution function that describes the range of dynamical properties of planetary systems. The observed distribution of $\beta$ is plotted in the bottom panel of Fig. 3. Most systems have $\beta \sim 1$, nearly all have $\beta<2$, and the exception is $\operatorname{HD} 217107(\beta>7)$. This clustering of systems near the Hill stability limit suggests that many systems form near the edge of dynamical stability.

In Fig. 3 and Table 1 we see that many systems in fact have $\beta<1$. These systems are all in mean motion resonance; the interaction is stabilized by the resonance. In fact, 
HD 108874 is the only known resonance system to have $\beta>1$. Therefore it seems that resonances tend to form such that $\beta<1$.

Table 1 Dynamical Properties of Multiple Planet Systems

\begin{tabular}{|c|c|c|c|c|c|c|c|}
\hline System & Pair & MMR & $\mathrm{AM}$ & $\epsilon$ & $\beta$ & Class & Reference \\
\hline $47 \mathrm{UMa}$ & $\mathrm{b}-\mathrm{c}$ & - & $\mathrm{C}^{a}$ & 0 & 1.025 & $\mathrm{~S}$ & Butler et al.(2006) \\
\hline \multirow[t]{3}{*}{$55 \mathrm{Cnc}$} & $e-b$ & - & $\mathrm{C}$ & 0.067 & - & $\mathrm{T}$ & Butler et al.(2006) \\
\hline & $\mathrm{b}-\mathrm{c}$ & $3: 1$ & $\mathrm{C}$ & 0.11 & - & $\mathrm{R}$ & \\
\hline & $c-d$ & - & $\mathrm{C}$ & 0.158 & - & $\mathrm{S}$ & \\
\hline GJ 317 & $\mathrm{~b}-\mathrm{c}$ & $4: 1 ?$ & $?^{b}$ & - & 0.98 & $\mathrm{R} ?$ & Johnson et al.(2007) \\
\hline \multirow[t]{2}{*}{ GJ 876} & $\mathrm{~d}-\mathrm{c}$ & - & $\mathrm{C}^{a}$ & 0 & - & $\mathrm{T}$ & Butler et al.(2006) \\
\hline & $c-b$ & $2: 1$ & $\mathrm{~A}$ & 0.34 & - & $\mathrm{R}$ & \\
\hline \multirow[t]{2}{*}{ Gl 581} & $\mathrm{~b}-\mathrm{c}$ & - & $\mathrm{C}$ & 0.15 & - & $\mathrm{T}$ & Udry et al.(2007) \\
\hline & $\mathrm{c}-\mathrm{d}$ & - & $\mathrm{C}$ & 0.20 & - & $\mathrm{T}$ & \\
\hline HD 12661 & $\mathrm{~b}-\mathrm{c}$ & - & $\mathrm{C}$ & 0.003 & 1.199 & $\mathrm{~S}$ & Butler et al.(2006) \\
\hline \multirow[t]{2}{*}{ HD 37124} & $\mathrm{~b}-\mathrm{c}$ & - & $\mathrm{C}$ & 0.009 & - & $\mathrm{S}$ & Butler et al.(2006) \\
\hline & $c-d$ & - & $\mathrm{A}$ & 0.096 & - & $\mathrm{S}$ & \\
\hline HD 38529 & $\mathrm{~b}-\mathrm{c}$ & - & $\mathrm{C}$ & 0.44 & 2.070 & $\mathrm{~S}$ & Butler et al.(2006) \\
\hline \multirow[t]{2}{*}{ HD 69830} & $\mathrm{~b}-\mathrm{c}$ & - & $\mathrm{C}$ & 0.095 & - & $\mathrm{T}$ & Lovis et al.(2006) \\
\hline & $c-d$ & - & $\mathrm{C}$ & 0.04 & - & $\mathrm{S}$ & \\
\hline $\mathrm{HD} 74156^{c}$ & $\mathrm{~b}-\mathrm{c}$ & - & $\mathrm{C}$ & 0.36 & 1.542 & $\mathrm{~S}$ & Butler et al.(2006) \\
\hline HD 73526 & $\mathrm{~b}-\mathrm{c}$ & $2: 1$ & $\mathrm{AA}$ & 0.006 & 0.982 & $\mathrm{R}$ & Butler et al.(2006) \\
\hline HD 82943 & $\mathrm{~b}-\mathrm{c}$ & $2: 1$ & $\mathrm{C}$ & 0.004 & 0.946 & $\mathrm{R}$ & Butler et al.(2006) \\
\hline HD 128311 & $\mathrm{~b}-\mathrm{c}$ & $2: 1$ & $\mathrm{C}$ & 0.091 & 0.968 & $\mathrm{R}$ & Butler et al.(2006)? \\
\hline HD 108874 & $\mathrm{~b}-\mathrm{c}$ & $4: 1$ & $\mathrm{C} / \mathrm{AA}^{d}$ & 0.2 & 1.107 & $\mathrm{R}$ & Butler et al.(2006) \\
\hline HD 155358 & $\mathrm{~b}-\mathrm{c}$ & - & $\mathrm{AA}$ & 0.21 & 1.043 & $\mathrm{~S}$ & Cochran et al.(2007) \\
\hline HD 168443 & $\mathrm{~b}-\mathrm{c}$ & - & $\mathrm{C}$ & 0.22 & 1.939 & $\mathrm{~S}$ & Butler et al.(2006) \\
\hline HD 169830 & $\mathrm{~b}-\mathrm{c}$ & - & $\mathrm{C}$ & 0.33 & 1.280 & $\mathrm{~S}$ & Butler et al.(2006) \\
\hline HD 190360 & $c-b$ & - & $\mathrm{C}$ & 0.38 & 1.701 & $\mathrm{~T}$ & Butler et al.(2006) \\
\hline HD 202206 & $\mathrm{~b}-\mathrm{c}$ & $5: 1$ & $\mathrm{C}$ & 0.096 & 0.883 & $\mathrm{R}$ & Butler et al.(2006) \\
\hline HD 217107 & $\mathrm{~b}-\mathrm{c}$ & - & $\mathrm{C}$ & 0.46 & 7.191 & $\mathrm{~T}$ & Butler et al.(2006) \\
\hline Hip 14810 & $\mathrm{~b}-\mathrm{c}$ & - & $\mathrm{AA}$ & 0.05 & 1.202 & $\mathrm{~T}$ & Wright et al.(2007) \\
\hline \multirow[t]{3}{*}{ SS } & $\mathrm{J}-\mathrm{S}$ & - & $\mathrm{C}$ & 0.19 & - & $\mathrm{S}$ & JPL \\
\hline & $\mathrm{S}-\mathrm{U}$ & - & $\mathrm{C}$ & 0.006 & - & $\mathrm{S}$ & \\
\hline & $\mathrm{U}-\mathrm{N}$ & - & $\mathrm{C}$ & 0.004 & - & $\mathrm{S}$ & \\
\hline \multirow[t]{3}{*}{$\mu$ Ara } & $c-d$ & - & $\mathrm{C}$ & 0.002 & - & $\mathrm{T}$ & Pepe et al.(2007) \\
\hline & $\mathrm{d}-\mathrm{b}$ & $2: 1$ & $\mathrm{C}$ & 0.003 & - & $\mathrm{R}$ & \\
\hline & b-e & - & $\mathrm{C}$ & 0.13 & - & $\mathrm{S}$ & \\
\hline \multirow[t]{2}{*}{$v$ And } & $\mathrm{b}-\mathrm{c}$ & - & $\mathrm{C}$ & $1.8 \times 10^{-4}$ & - & $\mathrm{T}$ & Butler et al.(2006) \\
\hline & $c-d$ & - & $\mathrm{C}$ & $2.8 \times 10^{-4}$ & - & $\mathrm{S}$ & \\
\hline
\end{tabular}

${ }^{a}$ The current eccentricity of one planet is 0, placing the pair on an apsidal separatrix.

${ }^{b}$ The values of mean longitude and longitude of periastron are unknown.

${ }^{c}$ These values do not incorporate the new planet Bean et al.(2007)

${ }^{d}$ This pair alternates between circulation and anti-aligned libration.

One noteworthy system in this context is GJ 317 (Johnson et al.2007), with a $\beta$ value of 0.98 (see Table 1). This value is only permissible if the system is in a mean motion 
resonance, and, indeed, the ratio of the periods is near a whole number (4.02). Usually a mean motion resonance is identified by calculating the "resonant argument", which depends on mean longitude and longitude of periastron (see, e.g. Murray \& Dermott 1999). However, in the case of GJ 317, these two angles are unknown, and, hence, the resonant argument is also unknown. Nonetheless, the system must be in resonance (barring substantial revisions in the masses, semi-major axes and eccentricities of the planets) based on our stability analysis. Therefore consideration of proximity to instability in terms of the Hill stability boundary may provide an alternative method for identifying mean motion resonances. As the orbital parameters of the two planets in GJ 317 are refined, we predict that they will, in fact, be found to be in resonance.

\section{Conclusions}

We have described here new approaches for parameterizing the dynamical interactions of extrasolar planet systems. Many systems interact such that they are near the stability limit and the apsidal separatrix. Most mean-motion resonance interactions would be unstable if not for the resonance, a feature which may be used to identify resonant interactions (e.g. GJ $317 \mathrm{~b}$ and c). The distribution of $\epsilon$ shows that the scattering of approximately equal mass planets is unlikely to produce the observed distribution of apsidal behavior. The distribution of $\beta$ values shows that many systems formed near the limit of dynamical stability.

These results demonstrate the benefits of the consideration of the dynamical interactions of multiple planet systems, which may constrain models of planet formation. For example, as we discussed above, the proximity of so many systems to an apsidal separatrix $(\epsilon=0)$ suggests that "rogue planets" may have played a major role in scattering planets to higher eccentricities. The proximity of so many systems to the stability limit $(\beta=1)$ suggests many systems form in densely packed configurations. Moreover, consideration of planetary systems in these terms has revealed that our Solar System shares dynamical traits with the known multiple planet systems. Perhaps constraining planet formation models through dynamical properties, rather than observed orbital elements, will lead to a universal model of planet formation.

About half of planetary systems are multiple (Wright et al.2007), predictions of additional companions are being borne out (Barnes \& Raymond 2004; Raymond \& Barnes 2005; Bean et al.2008), and the current distribution of planet masses suggest there will be many planets with a mass equal to that of Saturn or less (Marcy et al.2005), i.e. below current detection limits. These three observations imply many multiple planet systems will be detected in the future. Hence characterizing extrasolar planet interactions will be a critical aspect of the study of planet formation for the foreseeable future.

\section{References}

Artymowicz, P. 1992. PASP 104, 679.

Barnes, R. \& Greenberg, R. 2006. ApJ 638, 478. (BG06a)

Barnes, R. \& Greenberg, R. 2006. ApJ 647, L153. (BG06b)

Barnes, R. \& Greenberg, R. 2006. ApJ 652, L53. (BG06c)

Barnes, R. \& Greenberg, R. 2007. ApJ 659, L53. (BG07a)

Barnes, R. \& Greenberg, R. 2007. ApJ 665, L67. (BG07b)

Barnes, R. \& Quinn, T.R. 2001. ApJ 550, 884.

Barnes, R. \& Quinn, T.R. 2004. ApJ 611, 494.

Barnes, R. \& Raymond, S.N. 2004. ApJ 617, 569.

Bean, J. L. et al.2008. ApJ 672, 1202. 
Boss, A. P. 2000. ApJ. 536, L101.

Butler, R. P. et al.2006. ApJ 646, 505.

Chambers, J. E. 1999. MNRAS 304, 793.

Chiang, E. I. \& Murray, N. 2002. ApJ 576, 473.

Cochran, W. D. et al.2007. ApJ 665, 1407.

Correia, A. C. M. et al.2005. A\&A 440, 751.

Cuntz, M. et al.2007. ApJ 667, L105.

D'Angelo, G., Lubow, S. H. \& Bate, M. R. 2006. ApJ 652, 1698.

David, E. -M. et al.2003. PASP 115, 825.

Érdi, B. et al.2004. MNRAS 351, 1043.

Ford, E. B., Havlikova, M., \& Rasio, F. A. 2001. Icarus 150, 303.

Ford, E. B., Lystad, V. \& Rasio, F. A. 2005. Nature 434, 873.

Gladman, B. 1993. Icarus 106, 247.

Goldreich, P. \& Sari, R. 2003. ApJ 585, 1024.

Goździewski, K. 2002. A\&A 393, 397.

Hadjidemetriou, J. D. 2006. CeMDA 95, 225.

Holman, M. J. \& Wiegert, P. A. 1999. AJ 117, 621.

Kiseleva-Eggleton, L. et al.2002. ApJ 578, L145.

Laskar, J. 1990. Icarus 88, 266.

Laughlin, G. \& Adams, F. C. 1999. ApJ 526, 881.

Lin, D. N. C. \& Ida, S. 1997. ApJ 477, 781.

Lovis, C. et al.2006. Nature 441, 305.

Malhotra, R. 2002. ApJ 575, L33.

Marchal, C. \& Bozis, G. 1982. CeMDA 26, 311.

Marcy, G. W. et al.2005. ApJ 619, 570.

Marzari, F. \& Weidenschilling, S. 2002. Icarus 156, 570.

Michtchenko, T. A. \& Malhotra, R. 2004. Icarus 168, 237.

Milani, A. \& Nobili, A. M. 1983. CeMDA 31, 213.

Murray, C. D. \& Dermott, S. F. 1999. Solar System Dynamics. Cambridge UP, Cambridge.

Papaloizou, J. C. B., Nelson, R. P. \& Masset, F. 2001. A\&A 366, 263.

Pepe, F. et al.2007. A\&A 462, 769.

Quillen, A. C. \& Faber, P. 2006. MNRAS 373, 1245.

Rasio, F. A. \& Ford, E. B. 1996. Science 274, 954.

Rasio, F. A. et al.1996. ApJ 470, 1187.

Raymond, S. N. \& Barnes, R. 2005. ApJ 619, 549.

Raymond, S. N., Barnes, R. \& Kaib, N. A. 2006. ApJ 644, 1223.

Rivera, E. J. \& Lissauer, J. J. 2000. ApJ 530, 454.

Sándor, Zs. et al.2007. MNRAS 375, 1495.

Sosnitskii, S. P. 1999. AJ 117, 3154.

Stepinski, T. F., Malhotra, R. \& Black, D. C. 2000. ApJ 545, 1044.

Szebehely, V. \& McKenzie, R. 1981 CeMDA 23, 3.

Udry, S. et al.2007. A\&A 469, L43.

Voyatzis, G. \& Hadjidemetriou, J. D. 2006. CeMDA 95, 259.

Weidenschilling, S. \& Marzari, F. 1996. Nature 384, 619.

Wisdom, J. 1982 AJ. 87, 577.

Wright, J. T. et al.2007. ApJ 657, 533.

Zhou, J. -L. \& Sun, Y. -S. 2003. ApJ 598, 1290. 